\title{
Precursor phenomenon on TiNi R phase transformation by $\mathrm{X}$-ray diffraction
}

\author{
T. Ohba ${ }^{1, *}$ and Y. Miyata ${ }^{2, \dagger}$ \\ ${ }^{1}$ Department of Materials Science and Engineering, Teikyo University, Utsunomiya 320-8551, \\ Japan \\ ${ }^{2}$ Department of Environmental and Material Engineering, Teikyo University of Science and \\ Technology, Uenohara, Yamanashi 409-0193, Japan
}

\begin{abstract}
TiNi alloy system has been widely used for industrial application and fundamental research. Many studies were performed under circumstance that $R$ phase was not certainly established. Recently $R$ phase was established and structure of $\mathrm{R}$ phase was determined. Present study reports diffuse scattering study as a precursor phenomenon to R-phase by using four-circle diffractometer under circumstance that the structure was determined. Diffuse scattering was observed around $1 / 3$ of $[1 \overline{1} 0]^{*}$ incommensurate position at various temperatures in parent phase. Shift from commensurate position broke symmetry and temperature dependence looked random. FWHM of $1 / 3$ reflections and integrated intensities were measured also in parent phase. FWHM decreased and integrated intensities increased with approaching transformation temperature.
\end{abstract}

\section{INTRODUCTION}

TiNi is one of the most interesting and important alloy systems for both industrial uses and fundamental researches. Shape memory effect in TiNi is useful and widely used for industrial applications. Transformation process of TiNi system attracted many researchers from a fundamental aspect. Parent phase of TiNi has $\mathrm{B} 2(\mathrm{CsCl})$ type structure and transforms into monoclinic B19' martensite phase.[1,2] Addition of $\mathrm{Fe}$ changes transformation process and R-phase appears in $\mathrm{TiNi}(\mathrm{Fe})$ system.[3]

Although, R-phase is now understood to be a diffusionless and first-order transformation, there was discussion that the transformation was second-order transformation in previous time.[4] Though situation around $\mathrm{R}$ phase transformation was like that, mechanism of transformation attracted many researchers. Charge density model was proposed for transformation mechanism. [5] Hwang et al. [6] and Ling and Kaplow [4] measured electrical resistances of TiNi and reported that electrical resistance increased rapidly at $T_{R}$ and not obvious inflection point with decreasing temperature.

Not only transformation process to $R$ phase but also crystal structure of $R$ phase was not clear for long time. At the beginning of research, crystal structure of $R$ phase was considered to be obtained by rhombohedral distortion of parent cubic phase. Recently Hara et al. [7] determined crystal structure of $\mathrm{TiNi}(\mathrm{Fe}) \mathrm{R}$ phase with polycrystalline sample applying Rietveld method. They reported crystal structure of TiNi R phase was the same as AuCd $\zeta_{2}^{\prime}$ martensite.

Precursor study of transformation is important for understanding mechanism of the transformation. Diffusionless transformation led an attractive picture of transformation mechanism, that is, phonon softening. Phonon dispersion in parent phase was measured by Satija et al. [8] and Moine et al. [9]. They reported phonon softening at $\zeta=1 / 3$ of $[\zeta \zeta 0] \mathrm{TA}_{2}$ branch. Diffuse scattering study was also useful for the precursor study. Shapiro et al. [10] studied the diffuse scattering of premarutensite state. They reported diffuse scattering which appeared around $1 / 3$ of $[1 \overline{1} 0]^{*}$ as a precursor phenomenon. These $1 / 3$ reflections did not show any symmetry in Brillouin zone.

Since AuCd has similar martensite structure called $\zeta_{2}^{\prime}[11]$ and phonon softening around $\zeta=1 / 3$ of $[\zeta \zeta 0] \mathrm{TA}_{2}$ branch.[12], it is interesting to compare transformation process for complete understanding of transformation.[13] We studied precursor phenomenon measuring diffuse scattering appearing prior to R phase transformation. [5].

\footnotetext{
"Present address: Department of Materials Science, Shimane University, Nishikawatsu, Matsue 690-8504, Japan.

'Present address: The Graduate University of Advanced Studies, Tsukuba 305-0801, Japan.
} 


\section{EXPERIMENTAL PROCEDURE}

Ti-48at $\% \mathrm{Ni}-2 \mathrm{at} \% \mathrm{Fe}$ single crystals were grown with modified Bridgman method: The single crystal was spark cut into needle-shaped specimens with a size of approximately $1 \mathrm{~mm} \times 1 \mathrm{~mm} \times 50 \mathrm{~mm}$. These were heat-treated at $1273 \mathrm{~K}$ for $1 \mathrm{~h}$, and quenched into ice water. The needle-shaped specimens were electro-polished into small fragments with a solution which consist of hydrochloric acid: acetic acid $=1: 1$ in volume for X-ray diffraction experiments. The sizes of X-ray diffraction specimens were approximately $30 \mu \mathrm{m}$ in diameter. The small specimens were picked up and mounted on fiber shape glasses. Four-circle X-ray diffractometer at BL-10A in synchrotron radiation facility, Photon Factory (PF), in KEK and AFC-7R (Rigaku ) in NRIM(National Research Institute for Metals) were utilized for measurements. Utilized wavelength of PF was $1 \AA$ monochromated by $\operatorname{Si}(111)$ and that of NRIM was $0.71073 \AA, \mathrm{MoK} \alpha$. Imaging plate (IP) was utilized for determining transformation temperature in $\mathrm{PF}$ and NRIM. IP was placed at $2 \theta$ arm of four-circle diffractometer. Distance between IP and specimen was $180 \mathrm{~mm}$ and size of IP was $200 \times 250 \mathrm{~mm}$ in KEK and those were $75 \mathrm{~mm}$ and $127 \times 127 \mathrm{~mm}$ in NRIM, respectively. Oscillation photographs around $\langle 1 \overline{1} 0\rangle$ were taken and 110 Bragg reflection, which was placed in the equator, was exposed at various temperatures.

For precise measurements around $1 / 3$ reflections, two-dimensional mesh scans were employed. The measurements were made on $h-k$ reciprocal plane. Counting time for one step was $10 \mathrm{~s}$ or $40 \mathrm{~s}$ and interval were 0.005 or 0.01 in index for around $1 / 3$ reflection and $1 \mathrm{~s}$ and 0.01 for 110 Bragg reflections, respectively. Measurements were performed at several temperatures above Rs. Temperature was controlled by blowing cold $\mathrm{N}_{2}$ gas on the specimens.

\section{RESULTS AND DISCUSSION}

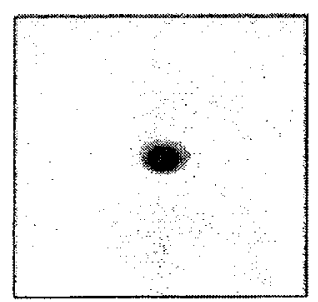

(a) $320 \mathrm{~K}$

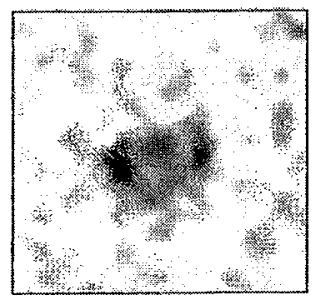

(c) $251 \mathrm{~K}$



(b) $252 \mathrm{~K}$

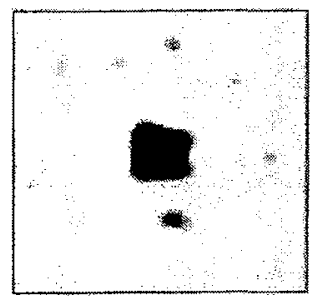

(d) $248 \mathrm{~K}$
Figure 1: Temperature dependence of 110 reflection exposed into IP. Transformation temperature was estimated to be $249 \mathrm{~K}$.
Figure 1 shows 110 Bragg reflection exposed at various temperatures. Bragg reflection without splitting was observed at $320 \mathrm{~K}$ and split into four Bragg reflections corresponding to variants of $R$ phase(Fig. 1(d)). Therefore, transformation temperature of R-phase (Rs) was determined to be $249 \mathrm{~K}\left(-24^{\circ} \mathrm{C}\right)$. Transformation temperature Rs used at NRIM was determined to be $242 \mathrm{~K}\left(-31^{\circ} \mathrm{C}\right)$ by the same method. Discrepancy came from method of measuring temperature. Temperature was monitored at approximately $5 \mathrm{~mm}$ from sample. Therefore, sample temperature was not measured directly.

Figure 2 represent results of two-dimensional, $h-k$ reciprocal plane around 1/3 5/3 0 , mesh scan measurements. Figure 2(a) to (e) represent contour intensity maps. Sharp reflections, which will be discussed below, were observed in Figure 2(a) measured at $275 \mathrm{~K}$. Diffuse scattering were observed in 


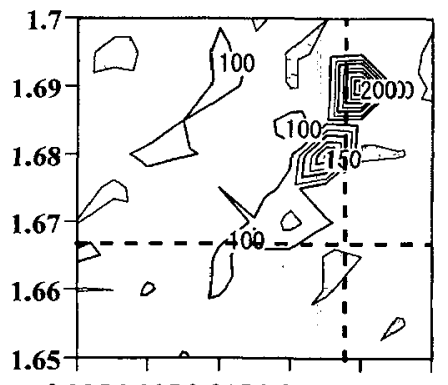

0.2950 .3050 .3150 .3250 .3350 .34 !

a) $275 \mathrm{~K}$

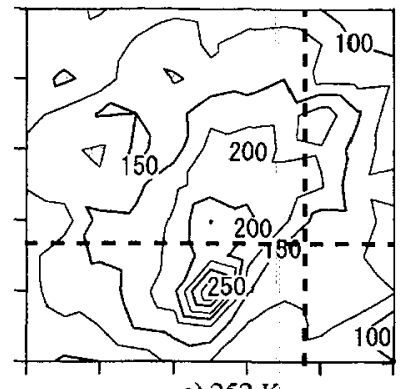

c) $252 \mathrm{~K}$

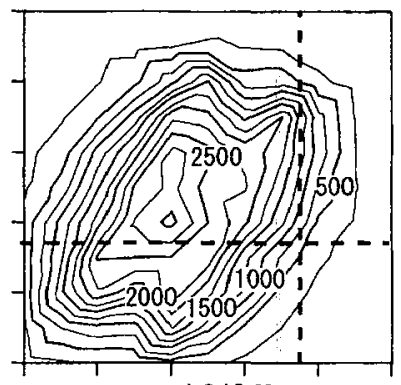

e) $248 \mathrm{~K}$



Figure 3: (a) Diffuse scattering observed around 1/3 position. Contour is drawn every $20 \mathrm{cps}$. Maximum intensity is approximately $180 \mathrm{cps}$.

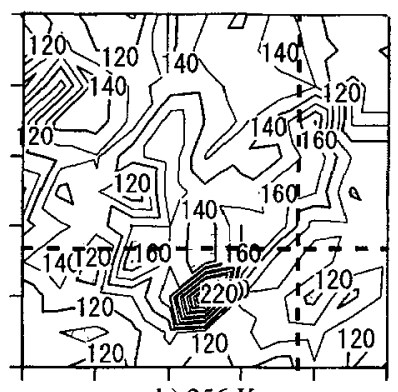

b) $256 \mathrm{~K}$

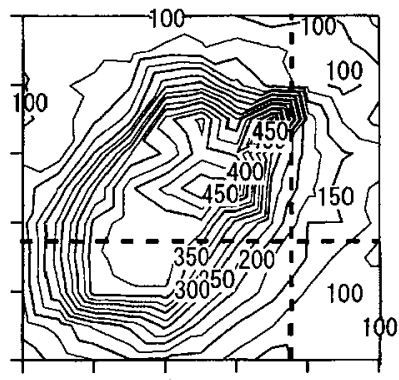

d) $250 \mathrm{~K}$

Figure 2: Temperature dependence of diffuse scattering at various temperatures. Transformation temperature was estimated to be $249 \mathrm{~K}$. Contours are drawn every 50cps. (e) represents reflection from $R$ phase. Maximum peak is approximately $2500 \mathrm{cps}$. lowering temperature (Fig. 2 (b) (d)). Figure 2(e) represents intensity distribution of R-phase reflection, which is consistent with observation of IP measurements, that is, four split shaped intensity was found. Peak intensity of martensite is about seven times stronger than that of diffuse scattering.

Position of $1 / 3$ reflection is important for discussing mechanism of the transformation. However, quality of beam at PF is much better than that of crystal itself. Therefore, sometimes reflections split reflected mosaic of the crystal, so lattice constant refined in this experiment was not satisfactory for discussing incommensurability reflections.

Figure 3(a), (b) represents contour map around 1/3 reflections at $\mathrm{Rs}+1 \mathrm{~K}$ observed in

NRIM. Lattice constant in this case were determined satisfactory and was $3.0147 \pm$ $0.0002 \AA$ in parent phase (room temperature). Therefore, position of $1 / 3$ reflection is discussed. Judging from observation, there is a shift from commensurate position shown in Fig. 3.

Shift from commensurate position of $1 / 3$ reflections are

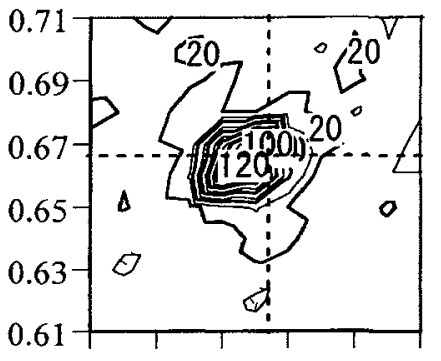



(b) Maximum intensity is approximately $140 \mathrm{cps}$. They were measured at $1 \mathrm{~K}$ above Rs. 


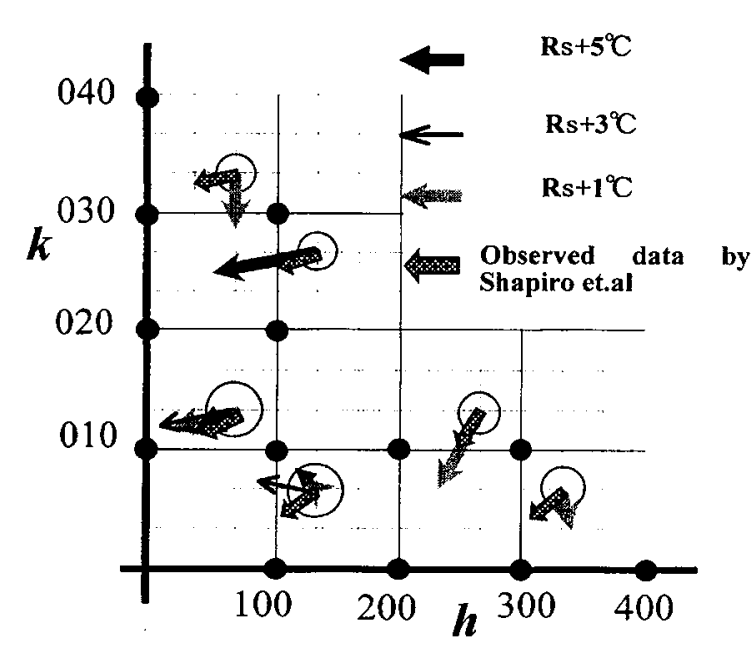

Figure 4: Shift of 1/3 reflections from commensurate positions and intensity distribution of present data. summarized in Fig. 4. Length of the arrows represent relative shift from commensurate position. Behavior from commensurate position is similar to previous report in a sense of non-periodicity but not completely the same.[10] Amount of shift was distributed around $0.007 \sim 0.02$ in index. Temperature dependence of shift was observed. They looked like random motion as shown in Fig. 4.

Integrated intensities at various temperatures were obtained from these observed data. They were plotted in Fig. 5. These were scaled at Rs+5 to compare with several data. Horizontal axis in this figure represents difference from Rs and vertical axis represents scaled integrated intensities. When temperature approached to $\mathrm{Rs}$ temperature, integrated intensities increased. Integrated

intensities shown above $\mathrm{Rs}+7 \mathrm{~K}$ do not show temperature dependence. These constant intensities must come from sharp extra reflections shown in Figure 2(a). They may be caused by something that is independent of transformation. One possibility of 'something' is reflections from impurities such as $\mathrm{Ti}_{2} \mathrm{Ni}, \quad \mathrm{Ti}_{2} \mathrm{NiH}_{0.5}, \mathrm{Fe}_{0.93} \mathrm{Ni}_{0.056}$ or $\mathrm{H}_{2} \mathrm{Ti}_{8} \mathrm{O}_{17}$.[14]

Diffuse scattering appeared prior to $\mathrm{R}$ phase transformation did not show features of $\mathrm{R}$ phase, that is, characteristic splitting shown in Fig. 2(e) was not observed. Without splitting reflection prior to $\mathrm{R}$ phase indicated that static products were produced as an intermediate or transitional state prior to the transformation temperature. The situation is similar to AuCd case. [13]

There are two possible interpretation of intensity increase, that is, volume of intermediate (transitional) state increased or number of intermediate state increased. When FWHM of Bragg reflections were measured, no remarkable change was observed. On the other hand, FWHM of $1 / 3$ reflections decreased with approaching transformation temperature. No remarkable change in Bragg reflections, which indicated the parent phase did not change drastically. Decrease of FWHM of $1 / 3$ reflections imply that volume of intermediate state increased.

Intensity distribution of $1 / 3$ reflections are also shown in Fig. 4 with open circles whose diameters are proportional to intensities.

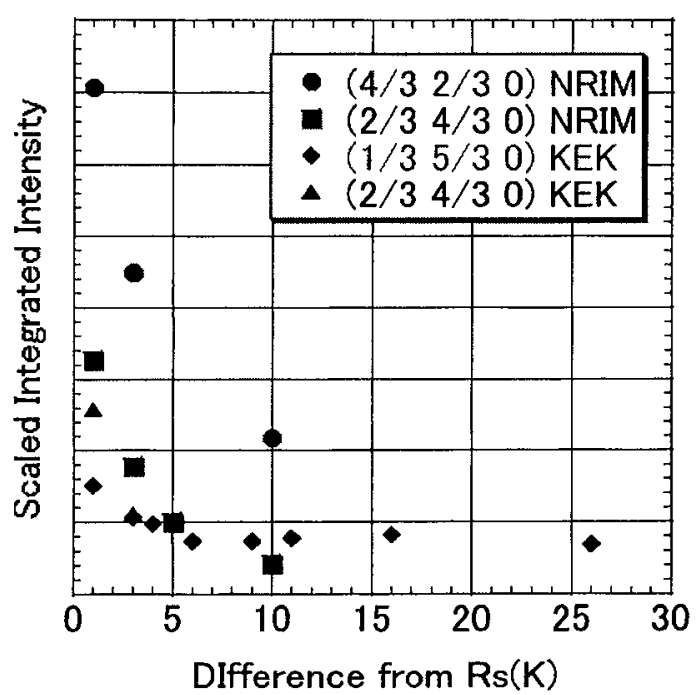

Figure 5: Temperature dependence of integrated intensities obtained from $1 / 3$ reflections. Intensities are scaled to 1 at Rs+5. KEK and NRIM represent place where experiments were performed. 
Intensities in low angle are stronger than higher angle reflections. There is a discrepancy between the previous report.[10] They reported intensities at higher angle reflections stronger than lower angle reflections and derived premartensitic phase structure. Calculation of structure factors of $R$ phase indicated that higher angle reflections are stronger than lower angle reflections. This means that premartensitic phase in previous studies were $\mathrm{R}$ phase itself. Diffuse scattering reported as precursor phenomenon seemed to come from $\mathrm{R}$ phase.

\section{CONCLUSIONS}

The diffuse scattering appearing prior to R-phase transformation in Ti-48at\% $\mathrm{Ni}-2 \mathrm{at} \% \mathrm{Fe}$ single crystal were measured by four-circle X-ray diffractometer at PF and NRIM. Diffuse scattering appeared around $1 / 3$ of $[1 \overline{1} 0]^{*}$ was considered a precursor phenomenon to R-phase transformation. Positions of the diffuse scattering are incommensurate and symmetry in Brillouin zone were broken and temperature dependence of the shift looked random. FWHM of 1/3 reflections were measured and they decreased with approaching transformation temperature. Integrated intensities of $1 / 3$ reflections were measured and increased with approaching Rs temperature.

\section{Acknowledgments}

The authors appreciate Professor Y. I. Chumlyakov of Tomsk University in Russia for preparing single crystal of TiNi(Fe). They also express their thanks to Dr. W. Cai(University of Tsukuba, now Harbin Institute of Technology in China) who prepared crystal used at NRIM. The authors are grateful to Dr. J. Ye (NRIM), Dr. M. Tanaka (PF, KEK) and Dr. K. Otsuka (National Institute for Advanced Interdisciplinary Research) for useful discussions. The authors are also wish to thanks for Messrs. J. Zhang, T. Ishii, S. Yamada, M. Inami and Miss N. Miura of University of Tukuba and K. Sato of Teikyo University for their assistant of the experiments.

\section{References}

1. R. F.Hehemann and G. D. Sandrock, Scripta metall. 5, 801 (1971).

2. K. Otsuka, T. Sawamura and K. Shimizu, Physica status solidi (a) 5, 457 (1971).

3. M. Matsumoto and T. Honma: Proc. Ist JIM Int. Symp. On New Aspects on Martensitic Transformations, Supplement to Trans. JIM, Vol. 17, 199 (1976).

4. H. C. Ling and R. Kaplow: Metallurgical Transactions 11A, 77 (1980).

5. M. B. Salamom, M. Meichle, C. M. Wayman, C. M, Hwang and S. M. Shapiro: Int. Conf. On Modulated Structures, Kona, Hawaii, AIP Conf. Proc. No. 53, 223 (1979), American Institute of Physics, New York

6. C. H. Hwang, M. Meichele, B. Salomon and C. M, Wayman: Philosophical Magazine A 47, 9, 31 (1983).

7. T. Hara, T. Ohba, E. Okunishi and K. Otsuka: Materials Transactions, JIM, 38, 11 (1997)

8. S. K. Satija, M. B. Salamom and C. M. Wayman: Physical Review, B, 29, 6031 (1984)

9. P. Moine, J. Allain and B. Renker: J. Phys. F: Met. Phys. 14, 2517 (1984)

10. S. M. Shapiro, Y. Noda, Y. Fujii, and Y. Yamada: Physical Review, B, 30, 4314 (1984)

11. T. Ohba, Y. Emura and K. Otsuka: Materials Trans. JIM, Vol. 33, 29 (1992).

12. T. Ohba, S.M.Shapiro, S. Aoki and K. Otsuka: Jpn. J. Appl. Phys. Vol. 33, L1631 (1994),

13. T. Ohba: Proc. of Int. Conf. on Solid-Solid Phase Transformations '99, 815 (1999).

14. Powder Diffraction File, JCPDS-International Center for Diffraction Data, Pennsylvania, U.S.A. 